\title{
New Definition for the Celestial Pole and the Celestial Origin in the ICRS
}

\author{
Nicole Capitaine \\ Observatoire de Paris, DANOF/UMR8630-CNRS, Paris, France
}

\begin{abstract}
Two Resolutions have been submitted to the $24^{\text {th }}$ General Assembly of the IAU concerning the definition and use of the celestial pole of reference and the celestial origin. The aim of both resolutions is to provide new parameters for Earth rotation which are consistent with the properties of the International Celestial Reference System (ICRS), adopted from 1 January 1998 as the IAU celestial reference system. The definition of the parameters have also to be consistent with the precision and the temporal resolution of the current Earth rotation measurements as well as with the theory for nutation and polar motion at the microarcsecond level. This paper explains the basis of the resolutions as well as their practical application. One of the resolutions defines the "Celestial Intermediate Pole" (CIP) in order to replace the "Celestial Ephemeris Pole" (CEP) for the new IAU precession-nutation model; its specifies the way for taking into account the constant offset from the ICRS and the high frequency terms in polar motion and nutation. The other resolution recommends the use of the "non-rotating origin" (Guinot 1979) on the moving equator, for defining Earth rotation and UT1; it also recommends the use of the celestial and terrestrial coordinates of the CIP in the transformation from the celestial to the terrestrial systems.
\end{abstract}

\section{Introduction}

The adoption of the International Celestial Reference System (ICRS) by the IAU since 1 January 1998 has provided a geocentric celestial reference frame for measuring Earth rotation which has no global rotation and is no more dependent on the Earth motion as was previously the FK5. Moreover, during the two last decades there has been a significant improvement in both the precision and the temporal resolution of the Earth rotation measurements as well as in the theory which now includes high frequency variations in the celestial nutation and in polar motion. This requires that the current definition of the Celestial Ephemeris Pole (CEP) be extended to the high frequency domain and that the Earth Orientation Parameters (EOP), which are currently defined in the FK5 System, be replaced by more basic parameters referred to the ICRS. Such issues have been under consideration by the subgroup T5 named "Computational Consequences" of the IAU Working Group "ICRS" whose preliminary proposals (see Capitaine 2000a and 2000b) have been largely discussed during IAU Colloquium 180 in Washington (March 2000) and have led to two reso- 
lutions adopted by the colloquium participants and submitted to the IAU for consideration and adoption during the XXIVth General Assembly. Resolution 7 "Definition of Celestial Intermediate Pole" provides an extended definition of the CEP in the high frequency domain and Resolution 8 "Definition and use of Celestial and Terrestrial Ephemeris Origin" provides new EOP for use in the coordinate transformation between the Geocentric Celestial Reference System (GCRS) and the Terrestrial Reference System (TRS) based on the adoption of the "non-rotating origin" (Guinot 1979) both with respect to the GCRS and the TRS for defining UT1.

\section{Definition of Celestial Intermediate Pole}

The coordinate transformation from TRS to GCRS makes use of an intermediate pole, the Celestial Ephemeris Pole (CEP), which is the current pole of reference for the IAU conventional models for precession and nutation. Since 1980 , there have been much improvement in the models for precession, nutation and polar motion as well as in processing observations of the EOP. Concerning the models, the semi-diurnal and diurnal prograde nutations, which were considered to be negligible, have been introduced in the solutions for nutation for a rigid Earth at a microarcsecond level since 1997 (Bretagnon et al. 1997). It must be noted that the diurnal prograde nutations in space are seen in the Earth as long periodic prograde and retrograde variations in polar motion and the semi-diurnal prograde nutations as prograde diurnal variations in polar motion (Bizouard et al. 2000). Similarly, the daily and subdaily tidal variations in polar motion (Herring and Dong 1994) have been included in the recent models for polar motion (McCarthy 1996). Concerning the observations, estimates of the corrections to the IAU precession and nutation (i.e. "celestial pole offsets") are published on a regular basis by the IERS since 1980; moreover, intensive EOP series are available and, due to their sampling interval smaller than one day, they do not allow a simultaneous estimation of nutation and polar motion of the CEP.

A new definition of the CEP has thus appeared to be necessary to be in agreement with modern models and observations and to take into account the overlapping between the motions in the GCRS and the TRS in the high frequency domain. The new pole which has been recommended, the "Celestial Intermediate Pole" (CIP) is such that its name emphasizes the fact that it is defined neither by a physical property, nor by a model, but that it appears as an intermediate pole in the coordinate transformation between the GCRS and the TRS. As the GCRS is not linked to the pole of J2000, the CIP has to be offset from the direction of the pole of the GCRS in a manner consistent with the IAU 2000 precession-nutation model. The CIP separates, by convention, the motion of the pole of the TRS (i.e. the pole of the mean surface geographic figure axis, or Tisserand mean axis of the Earth) into two parts: 1) the "celestial part," including the long period terms in the GCRS (i.e. the precession-nutation motion) with period greater than two days; 2) the "terrestrial part," including the whole complementary motion (i.e. the long periodic polar motion as well as the daily and sub-daily variations in polar motion); this part contains the variations in polar motion corresponding to the prograde diurnal and prograde 
semi-diurnal nutations as well as the daily and sub-daily tidal variations in polar motion. The motion of the CIP in the GCRS is realized by the IAU $2000 \mathrm{~A}$ model for precession and forced nutation for periods greater than two days plus the celestial pole offsets as estimated by observations. The motion of the CIP in the TRS is provided by EOP observations and takes into account a modelized part corresponding to the high frequency variations both in the GCRS and TRS. The corrections to this modelized part can be estimated using special procedures such as the one described by Mathews (1999).

\section{Definition and use of the Celestial and Terrestrial Ephemeris ori- gin}

The FK5 System is such that the equinox is the fundamental origin on the moving equator. The current precession angles are those defined by Lieske et al. (1977) in the FK5 System and the current nutation angles in longitude and in obliquity are referred to the ecliptic of date (Seidelmann 1982). The current procedure for taking into account precession and nutation is to use the matrix transformation corresponding to a sequence of six consecutive rotations based on five different parameters. As the precession and nutation angles are referred to the ecliptic of date, they are dependent both on the precession and nutation of the equator and on the precession of the ecliptic. The current procedure for taking into account Earth rotation in the FK5 System is to use the relationship between Greenwich Mean Sidereal Time, GMST, and UT1 (Aoki et al. 1982), followed by the relationship between Greenwich Sidereal Time, GST, and GMST. The difference GST-GMST is provided, since the 1st January 1997, by the "complete" equation of the equinoxes (Aoki and Kinoshita 1983; McCarthy 1996). Additionally to Earth rotation, the angle GST thus includes a part due to the accumulated precession (in GST) and nutation (in GMST) along the equator as well as a part (in GST-GMST) due to crossed terms between precession and nutation and crossed nutation terms. It refers to the ecliptic of date and thus mixes Earth's rotation and precession-nutation.

The fundamental properties of the ICRS, which has been adopted as the International Celestial Reference System (Ma et al. 1998), are the absence of global rotation and the abandonment of the link with the motion of the Earth. It is thus necessary to abandon the current parameters in the FK5 System which combine the motions of the equator and the ecliptic and to replace them by a minimal number of parameters, based on the location and motion of the rotation pole only without any relation to the orbital motion of the Earth. Consequently, such parameters must not use the equinox as the origin on the moving equator. Several options have been considered and compared (Capitaine 2000b), among which the most basic ones, the Euler angles between the CRS and the TRS and the celestial and terrestrial coordinates of the CIP. A comparison of the considered parameters shows that the Euler angles, or equivalently the celestial coordinates of the z-axis of the TRS, which reduce to three the number of EOP, do not use any intermediate pole. The Euler angles thus include both high frequency and low frequency components of the motion of the z-axis of the TRS in the CRS and their estimation from observations would need a large number of parameters. On the contrary, the celestial coordinates of the CIP separate the 
celestial components from the terrestrial components according to a "frequency criteria" using an intermediate pole. Such a procedure, in which the celestial pole coordinates appear in a symmetric form as the coordinates in the TRS, facilitates the estimation of the parameters from observations.

Several origins can also be considered on the moving equator for replacing the equinox. For defining an Earth's angle of rotation which includes only the "intrinsic Earth rotation", it is necessary to eliminate the spurious rotation of the origin on the moving equator due to the precession-nutation of the equator. A comparison of the alternative origins shows that, using a geometrical definition, the instantaneous rotation of the considered origin is included in the derived value for the Earth's angle rotation, whereas it is clearly separated when using an origin defined by the kinematical property of having "no instantaneous rotation" around the axis of rotation with respect to the GCRS. That provides the "non rotating origin", NRO (Guinot 1979), as the origin on the moving equator which can be designated as "the Celestial Ephemeris Origin" (CEO) if defined with respect to the GCRS. Similarly the "Terrestrial Ephemeris Origin" (TEO), designates the terrestrial NRO. The "stellar angle" is defined by the angle measured along the equator of the CIP between the CEO and the TEO and is such that UT1 is linearly proportional to this angle.

The coordinates of the CIP in the GCRS includes precession and nutation, the coupling effects between precession and nutation, as well as the offsets between the CIP at J2000.0 and the pole of the GCRS and between the mean equinox at J2000.0 and the origin on the equator of the CRS (Capitaine 1990). The development as function of time of the celestial coordinates of the CIP at a microarcsecond accuracy after one century are provided by Capitaine et al. (2000) in consistency with the IERS 1996 precession and nutation and the numerical values of the celestial offsets at J2000.0. Such developments can be easily modified to be consistent with the IAU 2000 precession-nutation model as soon as it is available.

The position of the CEO can be computed from the IAU 2000 model for precession and nutation of the CIP, whereas the position of the TEO is only slightly dependent on polar motion (Capitaine et al. 2000).

\section{Concluding remarks}

The IAU has adopted the International Celestial Reference System (ICRS) in replacement of the FK5 since the 1st January 1998. This adoption has been associated with significant improvement in the precision of Earth rotation measurements as well as in the theory of nutation and polar motion. The computational consequences for the consideration of Earth rotation are now taken into account through two resolutions to be adopted by the IAU at the XXIVth General Assembly in 2000. The resolution relative to the "Definition of Celestial Intermediate Pole" provides an extended definition of the CEP to the high frequency domain and the resolution relative to the "Definition and use of Celestial and Terrestrial Ephemeris Origin" abandons the current EOP, defined in the FK5 System, and replaces them by more basic parameters referred to the ICRS, providing a rigorous definition of the sidereal rotation of the Earth in the GCRS. 


\section{References}

Aoki S., Guinot, B., Kaplan G.H., Kinoshita, H., McCarthy, D.D. \& Seidelmann P.K. 1982, A\&A, 105, 359

Aoki, S. \& Kinoshita, H. 1983, Celest. Mech., 29, 335

Bizouard, C., Folgeira, M. \& Souchay, J. 2000, in Polar Motion: Historical and Scientific Problems, eds. S. Dick, D.D McCarthy \& B. Luzum, ASP, 613

Bretagnon, P., Rocher, P. \& Simon J.-L. 1997, A\&A, 319, 305

Capitaine, N. 1990, Celest. Mech. Dyn. Astr., 48, 127

Capitaine, N. 2000a, in Polar Motion: Historical and Scientific Problems, eds. S. Dick, D.D McCarthy \& B. Luzum, ASP, 573

Capitaine, N. 2000b, in Towards Models and Constants for Sub-microarsecond Astrometry, eds. K. Johnston, D.D. McCarthy, B. Luzum \& G. Kaplan, 153

Capitaine, N., Guinot, B. \& McCarthy, D.D. 2000, A\&A, 355, 398

Guinot, B. 1979, in Time and the Earth's Rotation, eds. D.D. McCarthy \& J.D. Pilkington, Reidel, 7

Herring, T.A. \& Dong, D. 1994, J. Geophys. Res., 99, 18051

Lieske, J.H., Lederle, T., Fricke, W. \& Morando, B. 1977, A\&A, 58, 1

Ma, C., Arias, E.F., Eubanks, M., Fey, A.L., Gontier, A.-M., Jacobs, C.S., Archninal, B.A. \& Charlot, P. 1998, AJ, 116, 516

Mathews, P.M. 1999, in Proc. of "Journées Systèmes de Référence SpatioTemporels" 1998, ed. N. Capitaine, Observatoire de Paris, 161

McCarthy, D.D. 1996, IERS Technical Note 21, Observatoire de Paris.

Seidelmann, P.K. 1982, Celest. Mech., 27, 79 